\author{
KATARZYNA BARBARA TYMOSZUK \\ Uniwersytet Marii Curie-Skłodowskiej w Lublinie \\ katarzyna.tymoszuk@umcs.lublin.pl \\ ORCID: 0000-0001-7801-200X
}

\title{
Kanal, Roboter, Geist, Eindringling, Chamäleon oder Mediator? Überlegungen zu den verschiedenen Rollen eines Kabinendolmetschers
}

\author{
Channel, robot, ghost, intruder, chameleon \\ or mediator? Various roles of the cabin interpreter
}

\begin{abstract}
The following paper is aimed at presenting and discussing the various roles - often mutually conflicting, or even unwanted - of the cabin interpreter. The starting point for the presented examples is an outline of the debate between practitioners and theoreticians of translation, centred around the actions that are expected from or prohibited for an interpreter in the situation of linguistic and cultural interpretation. The discussed concepts of roles are subsequently confronted with real-life examples of translator behaviours which have been taken from a broad research corpus obtained by the author.
\end{abstract}

KEYWORDS: simultaneous interpreting, interpreter's roles, interpreter competence, quality of interpretation.

SCHLÜSSELWORTE: Kabinendolmetschen, Dolmetscherrollen, Kompetenzen des Dolmetschers, Dolmetschqualität.

\section{ZIELSETZUNG}

Im vorliegenden Beitrag sollen die unterschiedlichen, nach außen hin selten wahrnehmbaren Rollen eines Kabinendolmetschers anhand authentischer Beispiele aufgezeigt und diskutiert werden. Den Ausgangspunkt bil- 
den die im translatorischen Diskurs schon immer widersprüchlichen Auffassungen darüber, welches Maß an Freiheit der in der Kabine agierende Translator überhaupt besitzt, wie frei er übersetzen darf, ggf. muss und wo ihm das - aufgrund welcher Faktoren - verboten ist. Mit anderen Worten: Welche Rolle(n) hat er einzunehmen? Hierbei werden sowohl die Fremdzuschreibungen seitens der Translationstheoretiker als auch die Selbstdarstellungen der Translatoren berücksichtigt.

\section{TRANSLATOREN - ERGIEBIGE METAPHERINSPIRATION}

Darf der Dolmetscher den Sprecher korrigieren? Sollte er sich notfalls bemerkbar machen? Inwieweit hat er überhaupt die Möglichkeit einzugreifen? In welchen Fällen sind bzw. wären seine Eingriffe als begründet und in welchen als fehlerhaft zu bewerten?

Der Begriff der Rolle, welche ein Translator in der sozialen Situation der vermittelten, zweisprachigen Kommunikation einzunehmen hat, steht seit eh und je im Zentrum des Interesses der Translationspraktiker und -theoretiker und die um dieses Thema wissenschaftlich-geführte Debatte kann gut und gerne verglichen werden mit dem Bild der Steinchenwerfer auf eine Wasseroberfläche und den dadurch ausgelösten konzentrischen Kreisen. Während die ersten, pragmatisch-sachlich-schlichten Erwägungen lediglich das Verhältnis des Translats zum Original betrafen (bezogen auf die Äquivalenzrelation, deren Herstellung das zentrale Ziel des Übersetzers ist), reflektierte man im Laufe der Zeit weitere wichtige Aspekte, die für den Kommunikationsverlauf ausschlaggebend und konstitutiv sind.

Dazu sollen im Folgenden die im Laufe der Zeit zu evaluierenden Rollenauffassungen des Translators kurz zugeordnet und dargestellt werden.

Die allererste Rolle eines zur Treue und Freiheit dem Ausgangstext gegenüber verpflichteten Sprachexperten (die gegenseitigen Positionen hierzu präsentierten bereits Cicero, Hieronymus von Stridon, Martin Luther und Vertreter verschiedener sprach- und übersetzungswissenschaftlicher Schulen; Strukturalismus, Stylistique Comparée, Leipziger Schule u.a.) (vgl. Prunč 2007) wurde dank der "pragmatischen Wende" neu definiert, und mit Publikationen zweier bahnbrechender finalistischer Translationsmodelle eingeleitet (vgl. Reiß \& Vermeer 1984; Holz-Mänttäri 1984). Ausschlaggebend dafür war die erweiterte Perspektive, aus welcher der gesamte Translationsprozess nun neu betrachtet werden sollte. Im Fokus der Aufmerksamkeit der Translationsleistungen stand fortan nicht mehr der Text allein - er wurde „entthront”, sondern auch die kommunikativen und situativen Faktoren 
in ihrer gänzlichen Komplexität ${ }^{1}$. Sie gewannen an Bedeutung und waren von nun an zu berücksichtigen. Das übergeordnete Ziel jeder Translation stellt von da an der Skopos dar. Für den Translator ist er die wichtigste Bezugsgröße bei der Wahl konkreter Translationshandlungen. Um den vom Redner angesetzten kommunikativen Effekt bei dem durch die Barriere der anderen Sprache abgegrenzten Zieladressaten zu erreichen, muss er zu einer Art von dritten Kommunikationspartner werden, indem er als Brückenbauer handelt. Nichtsdestotrotz dürfen seine Handlungen jedoch keinesfalls beliebig oder frei sein. Gewisse Einschränkungen werden den Handlungen des Translators durch das ethische Loyalitätsprinzip - ein von Christiane Nord Ende der 80er Jahre eingeführter Begriff (vgl. Nord 1989) - auferlegt. Loyalität soll dabei verstanden werden als „,...] die Bereitschaft und die Fähigkeit der Handelnden, die Interessen der übrigen HandlungspartnerInnen zur Kenntnis zu nehmen, zu reflektieren und angemessen darauf zu reagieren. Sie impliziert das gegenseitige Versprechen der HandlungspartnerInnen, nicht bewusst gegen die Interessen der anderen zu handeln" (Prunč 2009: 127). Der Translator soll dementsprechend die Rolle eines loyalen Handlungspartners übernehmen - und zwar in dreifacher Weise: gegenüber den Autoren, Auftraggebern und Zieltextempfängern (vgl. Nord 1989: 102).

Zur gleichen Zeit distanzierten sich auch die Forscher der literarischen Übersetzung, Antoine Berman und Lawrence Venuti, von der rein linguistischen Betrachtungsweise der Translationsprozesse. Sie sehen die Rolle des Translators, der sich des Fremden und der anderen Kultur bewusst ist, als Gärtner, der das zu übersetzende Werk aus seinen sprachlichen Wurzeln heran zieht, zugleich Entdecker ist und nach seinem innersten Kern sucht (vgl. Berman 1985; 2009) oder als mitverantwortlicher Zieltextgestalter, dessen Sichtbarkeit nicht selten durch die Strategie der foreignization / Verfremdung wünschenswert ist (vgl. Venuti 1995).

Noch einen Schritt weiter in seiner Interpretation der Rolle des Translators geht Erich Prunč (2009), der sie in Bezug auf den Begriff der Translationskultur definiert. Nach Prunč besteht eine Translationskultur „aus einem System von Normen und Konventionen, aber auch aus Wertvorstellungen, Erwartungshaltungen und habitualisierten Verhaltensmustern der AkteurInnen im jeweiligen sozialen und historischen Feld der Translation" (Prunč 2009: 121f). In diesem Kontext werden Translatoren, die im Machtspiel zwischen Autoren, Initiatoren und Adressaten immer geringere Rollen zu spielen scheinen, mit verschiedenen Chamäleonarten verglichen. Sie können sich, wie die meisten Chamäleons, der Mimesis bedienen und mit ihrer Um-

${ }^{1}$ Diese Aspekte wurden freilich auch schon von der Leipziger Schule, z.B. Otto Kade (vgl. Kade 1980) behandelt. 
gebung bis zur Unsichtbarkeit verschmelzen und sich auf diese Weise von jeglicher Interpretation distanzieren. Sogar von der Tarnmethode der Thanatose können sie Gebrauch machen, das heißt "sich tot stellen” (Prunč 2009: 125), um sich Interpretationszwänge oder kleinliche Besserwisser vom Leibe zu halten. Doch es steht ihnen auch die Möglichkeit zu, falls sie mit der gegebenen Translationskultur nicht einverstanden sind, „ihre Gegner sogar durch Aufreißen des Mauls" (Prunč 2009: 125) abzuschrecken oder (wie ein Jemenchamäleon) "gut hörbare Zischlaute von sich zu geben" (Prunč 2009: 125) und auf diese Weise den Widerstand gegen ihr Umfeld zu leisten. Somit können sie die verordneten Normen und Konventionen in Frage stellen oder sogar die gegebene Translationskultur beeinflussen.

Eine mit der Entwicklung der Translationswissenschaft einhergehende, immer umfassendere Betrachtungsperspektive der Rolle des Translators als unverzichtbarer Bestandteil des Translationsprozesses resultierte in einer ganzen Fülle an metaphorischen Rollenzuschreibungen. In einer seiner Vorlesungen ordnet Edward Balcerzan diese Menge an Rollenmetaphern und unterscheidet dabei solche, die an die Kunst oder Moral des Translators anknüpfen, wie: Verräter, Liebhaber, Rivale, Stratege oder Pianist, Chirurg und Porträtmaler (vgl. Balcerzan 2005: 42f.) und solche, die ihn als Ausführer sozialer Rollen darstellen, wie: Handwerker, Fahrer, Schauspieler, Musiker, Kämpfer u.v.m. Dabei betont der Forscher, dass die zu didaktischen oder propagandistischen Zwecken gebildeten Rollenmetaphern grundsätzlich einen zweifachen Charakter aufweisen. Entweder seien sie axiologisch und greifen damit übliche Stereotype oder Aphorismen auf, so dass die Translation wie eine Frau; entweder schön oder treu" sei und der Translator somit häufig ein "Verräter" "traduttore - traditore" ist. Die andere Gruppe von Metaphern entspringe der Epistemologie und suche für sich Argumente im bereits vorliegenden, erworbenen Wissen über das Wesen der Translation. Ein Beispiel hierfür sei die Metapher eines „Tänzers oder Akrobaten mit gefesselten Füßen”, was die überwältigende Mühe der translatorischen Arbeit veranschaulichen soll.

\section{SIMULTANDOLMETSCHER - ENTHUMANISIERTE HOCHSPEZIALISTEN}

Während jedoch die Tätigkeit eines Übersetzers schon seit langem als eine facettenreiche Kunst wahrgenommen und so auch in zahlreichen Rollenzuschreibungen widerspiegelt wird, wurde die Rolle des Dolmetschers lange Zeit missachtet. Es gab zwar Auffassungen, nach denen die Tätigkeit der Dolmetscher genauso anspruchs- und kunstvoll wie die der Übersetzer einzuschätzen ist. Von Herbert werden sie als Helfer oder Assistenten bezeichnet: 
"The interpreter should never forget that the immediate and essential object of his work is to enable his audience to know accurately what the speaker intended to convey, and to make on the audience the impression which the speaker wishes to be made" (Herbert 1952: 23). Andere, wie Cary (1956: 147) und Seleskovitch (1968: 181) vergleichen sie mit Schauspielern, die sich mit dem Redner vollständig identifizieren und in seine Haut schlüpfen müssen. Doch diese wenigen Ausnahmen lassen nicht die Tatsache übersehen, dass die Dolmetscher, vor allem Simultandolmetscher, lange Zeit beinahe enthumanisiert wurden. Manchen Forschern zufolge bestehe ihre einzige Aufgabe darin, Texte in emotionsloser, fast maschineller Art in die jeweilige Zielsprache zu übersetzen (vgl. Knapp-Potthoff \& Knapp 1986: 152). Der Grund einer solchen „mechanischen Konzeption” liegt gemäß Pöchhacker (2004: 147) sehr wahrscheinlich in der technologiegestützten Ausführungsweise des Dolmetschmodus und brachte seinerzeit eine ganze Reihe von Rollenmetaphern mit sich, von denen die des "treuen Echos" oder des „Kanals” ziemlich diplomatisch seien im Vergleich zum „Input-Output-Roboter", „Schaltgerät” oder „Treibriemen" (vgl. Pöchhacker 2004: 147). Solche Auffassungen verwundern umso mehr, wenn man sich vor Augen hält, dass die oben erwähnten Metaphern die "spektakulärste Erscheinungsform des Dolmetschens" (Pöchhacker 1998: 301) und einen der stressigsten Berufe überhaupt, gleich hinter den Berufen des Jetpiloten und Fluglotsen angereiht, bezeichnen. Doch die Geringschätzung der der Rolle des Kabinendolmetschers vertreten nicht nur Forscher und Theoretiker, sondern auch Auftraggeber und Kunden.

Die von Andrzej Kopczyński (1994: 90) durchgeführte Studie zeigt ebenso deutlich, dass sich auch Redner häufiger eine unpersönliche, möglichst zurückhaltende Haltung der in den Kabinen agierenden Translatoren wünschen. Kopczyńskis umfassende, die Qualität des Kabinendolmetschens im Allgemeinen betreffende Studie befragt u.a., ob der Dolmetscher aktiv in die Kommunikation eingreifen und somit die Rolle des Eindringlings (intruder role) übernehmen - oder wie ein Geist (ghost role) unsichtbar bleiben, d.h. keine Fragen stellen, nichts auslassen, hinzufügen oder zusammensetzen soll. Die Umfrage ergab, dass sich sowohl Nutzer als auch Redner für die Geisterrolle entschieden und: "the interpreter [...] should imitate the tempo and the intensity of voice of the speaker, but not necessarily the gestures" (Kopczyński 1994: 96). Ob die Simultandolmetscher mit diesen Rollenzuschreibungen einverstanden sind und wie sie selbst ihre Rolle im Prozess des Kabinendolmetschens betrachten, wurde in einer umfassenden Studie von Franz Pöchhacker und Cornelia Zwischenberger (2010) verifiziert. 704 AIIC-Mitglieder ${ }^{2}$ haben

${ }^{2}$ AIIC - ist die Abkürzung für die französische Bezeichnung des Internationalen Konferenzdolmetscherverbandes (Association internationale des interprètes de conférence). 
sich im dritten Teil der Umfrage zu Fragen über die eigene Rolle im Kommunikationsprozess geäußert. Die meisten betonten die eigene, helfende Funktion in solchen Metaphern, wie z.B. die des Kommunikationshelfers oder -begleiters (facilitator / enabler of communication). Andere rückten die Vermittlerfunktion ins Zentrum und nannten sich Mediatoren oder Brücken (mediator, bridge / link) und für eine weitere Gruppe betraf es die kommunikative Komponente - geäußert in Bezeichnungen wie die des Kommunikators oder des Kommunikationsexperten (communicator, communication_service / expert). Interessanterweise folgt aus der Studie auch die Zustimmung für Eingriffe in den Ausgangstext - ganz im Sinne Kopczyńskis - ergo für die Rolle des Eindringlings, welche mit dem Alter und der Arbeitserfahrung im Prestige steigt (vgl. Pöchhacker \& Zwischenberger 2010).

Dass eine solche Auffassung auch heute unter polnischen Vertretern des Berufes präsent ist, zeigt beispielsweise der in der neuesten polnischen Publikation zur Dolmetschdidaktik (vgl. Chmiel \& Janikowski 2015) präsentierte Beitrag von Zbigniew Nadstoga. In seinem Text über die Verhaltensnormen in der Dolmetschkabine listet der erfahrene Konferenzdolmetscher und Dolmetschdidaktiker alle möglichen Verbote auf: So darf der Simultandolmetscher nichts am Ausgangstext ändern, nichts hinzufügen, keine eigenen Reaktionen, Stellungsnahmen oder Gefühle andeuten, keine komplizierten Begriffe verwenden oder auch nicht im unangemessen, scherzhaften Ton in offiziellen Situationen sprechen. Nadstoga (2015) betont aber auch, dass das Register des zielsprachlichen Kommunikats immer an das Register des Ausgangssprechers anzupassen ist (vgl. Nadstoga 2015: 376f). Doch trotz dieser generellen Präferenz für die Geisterrolle lässt der Autor im weiteren Teil seiner Ausführungen auch ausnahmsweise die Rolle des Eindringlings oder sogar im Sinne von Prunč - die eines Jemenchamäleons zu. Falls nämlich die irritierten Kommunikationspartner in ihren zu dolmetschenden Reden zu vulgären Wörtern oder Wendungen greifen und der Dolmetscher sich damit nicht identifizieren will, kann er gewählte Fragmente mit entsprechendem Kommentar versehen oder seine Distanziertheit sogar in veränderter, grammatischer Form, der dritten Person Singular, manifestieren.

Die letzte, aus kommunikativer Perspektive ziemlich nebensächliche, doch im Kontext der Berufspraxis der Kabinendolmetscher zentrale Instanz, stellen die Translationsfirmen dar, die Auftragsvermittler der Translatoren also die direkten Auftraggeber - sind. Um festzustellen, wie man die Rolle der Kabinendolmetscher aus dieser Perspektive betrachtet und darstellt, reicht es u.U., ein paar Internetseiten oder Angebotsschreiben anbietender Firmen von Kabinendolmetschern zu analysieren. Häufig begegnet man Zusicherungen, dass die Unternehmen über die modernste Ausstattung 
verfügen und ein Kabinendolmetschen von höchster Qualität gewährleistet wird. Qualifikationen, Kompetenzen und Erfahrung der Dolmetscher werden dabei leider gar nicht erwähnt.

\section{BEISPIELE AUS DER PRAXIS}

Die obige Darstellung kann durchaus Zweifel nach sich ziehen, ob die präsentierte, vorwiegend theoretische Diskussion noch viel mit der Praxis zu tun hat und ob Kabinendolmetscher womöglich mit Situationen konfrontiert werden, die sie nicht selten in gegensätzliche Rollen zwingen.

Im weiteren Teil dieses Beitrags soll deshalb an gewählten Beispielen aus der translatorischen Praxis gezeigt werden, dass sich die Situationen einer zweisprachigen vermittelten Kommunikation in keinerlei Weise von anderen, einsprachigen Kommunikationssituationen unterscheiden. Dem Dolmetscher können in seiner beruflichen Funktion genauso viele Überraschungen begegnen wie in jeder anderen authentischen Lebenssituation, was ihn nicht nur zu ununterbrochener Aufmerksamkeit, sondern auch zur größtmöglichen Flexibilität und zu unerwarteten Rollenwechseln veranlasst.

Die gewählten Beispiele entstammen einem umfassenden Korpus von textuellen Modifikationen, welche vom jeweiligen Simultandolmetscher absichtlich oder unbewusst am Ausgangstext vorgenommen wurden. Als Basis für die Erarbeitung des Korpus (vgl. Tymoszuk 2016) diente das im Laufe von zwölf authentischen deutsch-polnischen simultan gedolmetschten Konferenzen erhobene Forschungsmaterial, das zunächst in Form von Tonaufnahmen eine Länge von ca. fünfzehn Stunden aufwies und nachfolgend nach extra entwickeltem Schlüssel (vgl. Tymoszuk 2016: 140ff.) transkribiert wurde. Den transkribierten Texten wurden alle Eigenschaften der Schriftlichkeit entzogen, indem sie in Versalbuchstaben und ohne alle für die schriftlichen Texte typischen Interpunktionszeichen gesetzt wurden. Ein Punkt wurde für den intonatorischen Abschluss des Satzes und für die leeren Sprechpausen verwendet. Die einzelnen Wörter und Silben, beabsichtigte und unbeabsichtigte Silbenverschmelzungen, Versprecher und unterbrochene Sequenzen wurden orthographisch transkribiert. Intonation und andere akustische und artikulatorische Erscheinungen wurden mit entsprechenden graphischen Symbolen markiert ${ }^{3}$. Die in den unten angegeben Beispielen befindlichen Symbole und Abkürzungen bedeuten entsprechend:

\section{AT - Ausgangstext \\ ZT - Zieltext}

\footnotetext{
${ }^{3}$ Mehr zum Transkriptionsverfahren und eine detaillierte Liste der Transkriptionszeichen wurden in Tymoszuk 2016 dargestellt.
} 
$\ni$ - gefüllte Pause/Zögern

[*] - Bemerkungen der dritten Personen (aller Personen außer dem aktuellen Sprecher)

Es würde von höchster Ignoranz und Unkenntnis des Dolmetsch-Alltags seitens der Autorin zeugen, zu behaupten, die Kabinendolmetscher wechselten ständig ihre Rollen und griffen in die zu dolmetschenden Texte unbegründet und eigenwillig ein. Die meiste Zeit bleiben sie der von allen Instanzen höchst akzeptierten Geisterrolle treu und liefern den Adressaten Texte, die den Ausgangskommunikanten in allen Parametern gleichen und dabei das Prinzip der Loyalität gegenüber allen Kommunikationspartnern nicht verletzen ${ }^{4}$. Doch wie bereits erwähnt, werden sie mitunter mit Situationen konfrontiert, in denen sie, um des allgemeinen Kommunikationserfolgs willen, kurz in die andere Haut "schlüpfen" müssen und so zu aller Art Eindringlingen werden.

\subsection{Experte}

Die am häufigsten anzutreffende Situation ist dabei mit der Expertenrolle verbunden. Unstrittig ist, dass kompetente Dolmetscher auch Sprachexperten sind oder sein müssen und zwar in Bezug auf beide in der jeweiligen Situation benutzten Sprachen. Die anderen Kommunikationspartner sind es dagegen meistens nicht, was nicht selten die Notwendigkeit sprachlicher Korrekturen an Ausgangstexten bedingt. So müssen die Translatoren zugleich auch ihr Sachwissen einbeziehen und die informativen Lücken oder Unklarheiten der Ausgangssequenzen ergänzen.

Die ersten zwei Beispiele zeigen, dass die informativen Ergänzungen in Form quantitativer Textexpansionen ${ }^{5}$ die Verständlichkeit ausgangssprachiger Kommunikate entscheidend erhöhen und somit eventuellen Unklarheiten im weiteren Kommunikationsverlauf vorbeugen.

B.1.

AT DIE MEINES ERACHTENS NICHT STICHHALTIG IST. ENTFÄLLT AUCH EIN WICHTIGES ARGUMENT DER ABGRENZUNG ZWISCHEN STAAT UND ZIVILGESELLSCHAFT. MACHT GIBT ES IN BEIDEN.

\footnotetext{
${ }^{4}$ Natürlich sind hier solche Dolmetscher gemeint, deren Kompetenzen, Kenntnisse und Qualifikationen es ihnen erlauben, das angestrebte Ziel bestmöglicher Dolmetschleistung zu erreichen.

${ }^{5}$ Die Begriffe der Expansionen und Kompressionen als die häufigsten qualitativen und quantitativen Modifikationen der Ausgangstexte im Prozess des Simultandolmetschens werden in Tymoszuk 2016 definiert und weiter klassifiziert.
} 
ZT WEDEUG MNIE NIE DA SIĘ TEGO UTRZYMAĆ ELIMINUJE WAŻNY ARGUMENT ODGRANICZENIA OD PAŃSTWA A WIĘC WŁADZA ISTNIEJE W OBU ZAKRESACH

B.2.

AT ICH DENKE DASS DAS BEISPIELSWEISE DIESE EREIGNISSE LEISTUNGEN SIND DIE AUCH EINMAL DIE WAHRNEHMUNG IN WESTEUROPA PRÄGEN

ZT SĄ TO RZECZYWIŚCIE ZDARZENIA KTÓRE ZMIENIAJĄ ZUPEŁNIE SPOSÓB POSTRZEGANIA TYCH KRAJÓW W EUROPIE ZACHODNIEJ

Das Gleiche wird in zwei weiteren Beispielen erreicht jedoch mit dem Unterschied, dass im Zieltext keine ergänzenden Elemente hinzugefügt werden, sondern die von dem Ausgangstextautor in der jeweiligen Situation wahrscheinlich sehr allgemein verwendeten Begriffe semantisch intensiviert, also präzisiert werden.

B.3.

AT WENN SIE DEUTSCHE Э BIBLIOTHEKSGESCHICHTE BEARBEITEN WERDEN SIE IMMER WIEDER AUF DIESE ANWEISUNG STOSSEN

ZT GDY BĘDZIECIE PAŃSTWO ZAJMOWALI SIĘ TĄ HIST- HISTORIĄ BIBLIOTEKARSTWA W NIEMCZECH CZĘTO BĘDZIECIE SPOTYKALI SIĘ Z INTRUKCJĄ 22 Z TYSIĄC DZIEWIĘĆSET SZEŚĆDZIESIĄT DZIEWIĘĆ.

B.4.

AT DZIEWIĘĆ KRAJÓW TEŻ JEST SPOZA EUROPY

\section{ZT NEUN LÄNDER AUßERHALB DER EUROPÄISCHEN UNION}

Das nächste Beispiel zeigt deutlich, wie unerlässlich es für den weiteren, erfolgreichen Kommunikationsverlauf ist, dass der Dolmetscher als Sprachund Sachexperte eingreift. Die Ausgangssequenz kennzeichnet nicht nur einen hohen Redundanzgrad. Sie ist syntaktisch inkohärent, informativ lückenhaft und stilistisch unangemessen. In dieser Situation muss der Dolmetscher den Text nicht nur stilistisch und syntaktisch korrigieren, sondern auch informativ ergänzen, indem er Informationen verbalisiert, die vom Sprecher lediglich angedeutet werden. Wäre er seiner üblichen Geisterrolle treu geblieben, wäre das unveränderte Textfragment für die Adressaten wahrscheinlich komplett unverständlich gewesen.

B.5.

AT NO ŻE PO PROSTU NAGLE SIĘ OKAZUJE ŻE SĄ JAKIEŚ QUASI TAKIE. NO JAKIEŚ NO SĄ JAKIEŚ ŻYCIORYSY NO NO I TO PO PROSTU JEST TYLKO TYLKO TYLE

ZT ES ZEIGT SICH PLÖTZLICH DASS ES HIER SO. Э SOLCHE LEBENSLÄUFE GIBT DIE DAZU NICHT PASSEN ALSO DAS WOLLTE ICH NUR SAGEN 


\subsection{Kommunikationshelfer}

Nicht selten zeigt sich die Rolle des Kommunikationshelfers für den reibungslosen Verlauf der Kommunikation als ausschlaggebend. In seinem Dolmetschalltag hat der Translator nicht nur mit Reden, Vorträgen und Berichten zu tun - also mit Texten, in denen die Sprachrichtung konstant bleibt und die den unilateralen Dolmetschmodus bedingen. Simultan gedolmetscht werden auch Podiumsdiskussionen, Fragen aus dem Publikum und andere Textkomplexe, in denen die Sprecher häufig wechseln und bilaterales Dolmetschen zum Einsatz gebracht wird. In solchen Situationen (vgl. Beispiel 6) kann das sogenannte Eindringen des Kabinendolmetschers in der Rolle des Kommunikationshelfers für die Hörer eine notwendige Orientierungshilfe darstellen.

B.6.

AT NO DOBRZE MOŻNA TO TAK OKREŚLIĆ ALE W TYM TAKIM Э Э ROZUMIENIU BARDZIEJ

ZT JA JA MAN KÖNNTE DAS SO BEZEICHNEN SAGT FRAU PROFESSOR. ALSO

Beispiele 7 und 8 zeigen wiederum, dass die Rolle des Eindringlings nicht immer mit den (qualitativen oder quantitativen) Expansionen der Ausgangstextsequenzen verbunden ist. Als Eingriff in den Ausgangstext wird auch das Weglassen von Elementen verstanden. Wenn weggelassene Wörter bzw. Wendungen nur den Redundanzgrad der Ausgangstextsequenz erhöht, steigert das Weglassen (die Kompression) auch entscheidend die Qualität der Kommunikation. Im Beispiel 7 verzichtet der Dolmetscher auf wiederholtes Dolmetschen zweier synonymer Bezeichnungen des gleichen Denotats, was keinerlei informative Verarmung der Sequenz verursacht, die Sprachökonomie jedoch wesentlich verstärkt. Zum gleichen Ergebnis führt das Beispiel 8 aufgrund des Weglassens von Füllwörtern.

B.7.

AT ICH HABE DIESE DIESES BUCH WIE GESAGT GEMEINT ALS EINE ANALYTISCHE BESCHREIBUNG ANALYTISCHE DESKRIPTION VERSCHIEDENER IDIOME DES THEATERS.

ZT KSIĄŻKA TA STANOWI DLA MNIE OPIS ANALITYCZNY RÓŻNYCH IDIOMÓW TEATRU.

B.8.

AT MAN KANN ALSO SAGEN DAS WAS THEATER IMMER SCHON WAR SOZUSAGEN DIESES SOZIALE EREIGNIS WIRD IM IM POSTDRAMATI- 
SCHEN THEATER SOZUSAGEN EIGENTLICH BETONT [.] AUF KOSTEN EINER EINER FIKTION [.]

ZT MOŻNA WIĘC POWIEDZIEĆ ŻE TO CZYM TEATR ZAWSZE BYモ CZYLI TO WYDARZENIE SPOŁECZNE W TEATRZE POSTDRAMATYCZNYM JEST PODKREŚLANE JEST ROZGRYWANE NA KOSZT PEWNEJ FIKCJI

\subsection{Mediator}

Die spezifischen, kulturellen Umstände und Anforderungen der jeweiligen Kommunikationssituation sollen grundsätzlich von allen Kommunikationsteilnehmern wahrgenommen, reflektiert und beachtet werden. In der Praxis sind jedoch ideale Mitglieder des Translationsgefüges nur sehr selten anzutreffen. Die realen, unvollkommenen Sprecher dagegen vergessen oder ignorieren manchmal kulturbedingte Regeln, was zu Konfliktsituationen führen kann. In ihrer Mediatorrolle beugen die Dolmetscher solchen Kontroversen auf unterschiedliche Weisen vor. Das neunte Beispiel zeigt den Austausch des alltäglichen und stilistisch neutralen Wortes być (de. sein) gegen eine situativ viel eher adäquate Formulierung ein Amt bekleiden.

B. 9.

\section{AT JEST ON DYREKTOREM INSTYTUTU \\ ZT $\ni$ BEKLEIDET ER NOCH DAS AMT DES DIREKTORS}

Die Beispiele 10 und 11 entstammen der gleichen Translationssituation und derselben Sprecherin, die ihren Vortrag völlig spontan (Idealsituation eines jeden Dolmetschers) gestaltet. Jedoch bleibt sie vorwiegend auf der umgangssprachlichen Ebene. Ihre Äußerung ist voller Kolloquialismen. Der Dolmetscher bleibt zwar die meiste Zeit seiner Rolle des loyalen Geistes treu - der Stil der Zieltextsequenzen ist auch relativ locker - doch in extrem kontroversen Fällen übernimmt er die Rolle des Mediators und ersetzt sehr umgangssprachliche und teilweise emotional negativ markierte Wendungen: czepiać się (de. stänkern) oder przelecieć się (de. ablatschen) durch standardsprachliche und bei den Hörern keine negativen Konnotationen hervorrufende Wörter: ersuchen und gehen.

B. 10 .

AT CZEGO DLACZEGO TRYBUNAE KONSTYTUCYJNY SIĘ TAK BARDZO CZEPIA

ZT WARUM ..... WAS ERSUCHT HIER DAS VERFASSUNGSGERICHTSHOF 
B. 11.

AT W WIELKI PIĄTEK PRZELECIAŁAM SIĘ PO SĄSIADACH ZEBRAŁAM POD LISTEM OSIEMDZIESIĄT PODPISÓW

ZT AM KARFREITAG BIN ICH Э BIN ICH ZU DER NACHBARIN GEGANGEN HAB ACHTZIG UNTERSCHRIFTEN GESAMMELT

Im zwölften Beispiel wird die unangemessene und kontroverse Stilistik zweifach: qualitativ und quantitativ neutralisiert. Das umgangssprachliche Wort idiotyczny (de. idiotisch) wird durch das Adjektiv furchtbar ersetzt, das zwar auch negative Konnotationen hervorruft, doch ein Mittel der Standardsprache und situativ angemessener ist. Das metaphorisch für das Schaffen eines neuen Parkplatzes auf einem Rasen gebrauchte Adjektiv wygryzio$n y$ (de. ausbeißen) wird dagegen ganz weggelassen, was jedoch zu keinem Informationsverlust führt, dagegen aber negative Konnotationen vermeidet.

B. 12.

AT POMYŚLAŁAM SOBIE OHO PEWNIE WŁAŚCICIEL DOMU PRZED KTÓRYM TO SIĘ DZIEJE CHCE SOBIE URZĄDZIĆ PARKING TAM I ŻE TO IDIOTYCZNIE WYGLĄDA BO TAKA DZIURA WYGRYZIONA W TYM TRAWNIKU.

ZT ICH HABE GEDACHT AHA DER EIGENTÜMER DES HAUSES DORT MÖCHTE DORT EINEN PARKPLATZ SICH WAHRSCHEINLICH ARRANGIERT DAS SIEHT FURCHTBAR AUS DAS IST EIN EIN LOCH Э DA IM RASEN

Als Manifestation der Mediatorrolle kann auch der Kommentar im Beispiel 13 angesehen werden. Zwar erklärt der Sprecher seinen Versprecher selbst, doch der Dolmetscher bleibt keinesfalls nur neutraler Geist. Mit seinem fast schon emotionalen Kommentar versucht er in noch stärkerem Maße als der Redner selbst, der Konfliktsituation vorzubeugen.

B.13.

AT FRAU IUGA UND ICH HABEN GESTERN SEHR INTERESSANT GESTORBEN

.. JA ALSO TROTZ EINES SEHR INTENSIVEN GESPRÄCHES LEBEN WIR NOCH. WIR HATTEN GESPROCHEN ÜBER DAS STERBEN VON WÖRTERN SO KAM MEIN VERSPRECHEN ZUSTANDE

ZT I TUTAJ PANI IUGA I JA WCZORAJ. UMARLIŚMY ALE TO PRZEPRASZAM BARDZO TO JEST WŁAŚNIE JĘZYK KIEDY SIĘ CIĄGLE ZMIENIAJĄ ... TO BYŁO RZECZYWIŚCIE TO BYŁA TAKA FREUDOWSKA POMYŁKA ... A WIĘC POMIMO TEJ INTENSYWNEJ ROZMOWY CIĄGLE JESZCZE ŻYJEMY ALE MÓWILIŚMY O UMIERANIU SŁÓW I STĄD AKURAT TA POMYŁKA TEN LAPSUS 


\subsection{Jemenchamäleon}

Die Metapher des Jemenchamäleons wurde zwar in Bezug auf den viel breiteren Kontext der Translationskultur entwickelt, doch ein gewisses "Aufreißen des Mauls" ist mitunter auch seitens der Kabinendolmetscher im jeweils kleinen Translationskontext unentbehrlich. Abgeschottet in einer oft in einem anderen Raum befindlichen Dolmetschkabine haben die Translatoren kaum oder so gut wie gar keinen Einfluss auf die gesamte Kommunikationssituation, falls sie ihrer Geisterrolle treu bleiben wollen. Vor allem in Situationen, in denen ihre Arbeit durch äußere Faktoren gefährdet wird, müssen sie verbal eingreifen. Andernfalls tragen ausschließlich sie die Verantwortung für eventuelle Missverständnisse, die infolge mangelhaften Dolmetschens zustande gekommen sind. Die erforderlichen "Zischlaute" in Form eigener Kommentare können als gewisse Entschuldigung für das Unvermögen, weiterhin hochwertige Dolmetschleistung zu gewährleisten, ans Publikum gerichtet werden (Beispiele 14 und 15).

B.14.

AT PONIEWAŻ I EDUKACJA III SŁUŻBA ZDROWIA I BUDOWNICTWO BYŁO UPAŃSTWOWIONE WIĘC TRUDNO TUTAJ MÓWIĆ ŻE SŁUCHAM .... NO DOBRZE MOŻNA TO TAK OKREŚLIĆ ALE W TYM TAKIM Э Э ROZUMIENIU TAMTEJSZYM PRAWDA Э Э

ZT ZU VERDANKEN DIE BILDUNG UND DIE GESUNDHEITSFÜRSORGE UND DAS WOHNBAUWESEN WAR STAATLICH ...\{ENTSCHULDIGUNG DIE DOLMETSCHER HABEN DAS JETZT GERADE NICHT GEHÖRT TUT MIR LEID DER HERR HAT NICHT INS MIKROPHON GESPROCHEN\}

B.15.

AT I CHCIAŁBYM NAJPIERW NA WSTĘPIE PARĘ SŁÓW PRZECZYTAĆ Э PIÓRA ANDRZEJA STASIUKA O RUMUNII JEGO LIEBESERKLAERUNG JEGO OŚWIADCZENIU MIŁOŚCI DO RUMUNII POWIEDZIAŁ TO W TEN SPOSÓB NIE TAK DAWNO W WYWIADZIE

ZT UND VIELLEICHT ERLAUBEN SIE MIR DASS ICH ZUNÄCHST VON ANDRZEJ STASIUK ETWAS VORLESEN WERDE ÜBER RUMÄNIEN DAS IST EINE LIEBESERKLÄRUNG VON STASIUK AN RUMÄNIEN ER HAT VOR KURZEM IN EINEM INTERWIEV FOLGENDES GESAGT \{WIR HABEN DEN TEXT LEIDER IN DER KABINE NICHT SAGT DIE DOLMETSCHERIN\}

Festgestellt wurden auch einige Fälle, bei denen sich Dolmetscher mit gewisser Mahnung an die Sprecher wendeten und sie zur Mikrophondisziplin veranlassten, wie es im unteren Beispiel 16 der Fall ist. 
B.16.

AT NA NA ZJEŹDZIE CDU CHYBA BYŁ CHYBA CHYBA BYŁ WTEDY [ XXX ] A KIRCHENTAG HABEN SIĘ RECHT ENTSCHULDIGUNG JA JA DIES IST DAS JA TO BYŁ TO BYŁ ZAJAZD ZJAZD KOŚCIOŁA [ XXX ] TERAZ PISAŁA TERAZ PISAŁA TE WSPOMNIENIA Э

ZT BEI EINEM PARTEITAG WAR DAS GENAU BEI EINEM KIRCHENTAG DAS WAR $\ni$.. \{INS MIKROPHON SPRECHEN BITTE FRAU PROFESSOR SCHWAN INS MIKROPHON \} NUN JETZT HAT SIE DIESE ERINNERUNGEN GESCHRIEBEN

Und letztlich schalten Dolmetscher auch die an die Kommunikation nicht direkt beteiligten Personen ein, wie z.B. technische Mitarbeiter oder andere Sprecher (vorausgesetzt, dass sie Kopfhörer tragen) in die Kommunikationssituation ein, welche als ihre physischen Repräsentanten die erforderlichen Korrekturen durchführen.

\section{B.17. \\ AT $[\ldots . . .$.$] IHRE FRAGE ANTWORTEND ALSO KANN ICH$ \\ ZT \{BARDZO PROSZE MIKROFON MIKROFON\} WIADAJĄC NA PAŃSKIE PYTANIE MUSZE POWIEDZIEĆ ŻE \\ NO CÓŻ ODPO-}

\section{FAZIT}

Unstrittig ist, dass jeder Kabinendolmetscher in seiner translatorischen Arbeit dem Ideal des Geistes möglichst nahe kommen soll. Diese These vertreten nicht nur die Translationstheoretiker. Sie wurde durch mehrere Studien, an denen Kunden, Adressaten und Dolmetscher teilnahmen, bestätigt. Doch, wie die obigen Beispiele beweisen, gibt es auch Momente, in denen das angestrebte Ideal Platz für eine gegensätzliche Rolle machen muss. $\mathrm{Ob}$ dabei die Rolle des Eindringlings als Experte, die des Helfers, Mediators oder sogar des umstrittenen Jemenchamäleons übernommen werden soll, bleibt natürlich der individuellen Situation und fachlichen Kompetenz geschuldet.

Die Beispiele zeigen jedoch mehr: Sie bestätigen, dass es eine Reihe von Rollenzuschreibungen gibt, die in Bezug auf Translatoren sicherlich nicht zutreffen. Gemeint sind an dieser Stelle v.a. sämtliche, mit der mechanischen Konzeption der Simultandolmetscherrolle verbundene Metaphern des Roboters, Kanals oder Schaltgerätes. 


\section{LITERATURVERZEICHNIS}

Balcerzan, E. (2005). Metafory, które „wiedzą”, czym jest tłumaczenie. Teksty Drugie, 5, 41-52. Berman, A. (1985, 2009). Przekład jako doświadczenie obcego. In: P. Bukowski / M. Heydel (Hrsg.), Wspótczesne teorie przektadu (S. 247-264). Kraków: Wydawnictwo Znak.

Cary, E. (1956). La Traduction dans le monde modern. Genève: Georg.

Chmiel, A. / Janikowski, P. (Hrsg.) (2015). Dydaktyka ttumaczenia ustnego. Katowice: Stowarzyszenie Inicjatyw Wydawniczych.

Herbert, J. (1952). The interpreter's handbook: How to become a conference interpreter. Genève: Georg.

Holz-Mänttäri, J. (1984). Translatorisches Handel. Theorie und Methode. Helsinki: Suomalainen Tiedeakatemia.

Kade, O. (1980). Die Sprachmittlung als gesellschaftliche Erscheinung und Gegenstand wissenschaftlicher Untersuchung. Leipzig: Enzyklopädie.

Knapp-Potthoff, A. / Knapp, K. (1986). Interweaving two discourses - the difficult task of the non-professional Interpreter. In: J. House / S. Blum-Kulka (Hrsg.), Analyzing intercultural communication (S. 181-211). The Hague: Mouton.

Kopczyński, A. (1994). Quality in conference interpreting: Some pragmatic problems. In: S. Lambert / B. Moser-Mercer (Hrsg.), Bridging the gap: Empirical research in simultaneous interpretation (S. 87-99). Amsterdam-Philadelphia: John Benjamins.

Nadstoga, Z. (2015). Tłumacz na rynku pracy. In: A. Chmiel / P. Janikowski (Hrsg.), Dydaktyka ttumaczenia ustnego (S. 365-384). Katowice: Stowarzyszenie Inicjatyw Wydawniczych.

Nord, Ch. (1989). Loyalität statt Treue. Vorschläge zu einer funktionalen Übersetzungstypologie. Lebende Sprachen, 14 (3), 100-105.

Pöchhacker, F. (1998). Simultandolmetschen. In: M. Snell-Hornby / H. G. Hönig / P. Kussmaul / P. A. Schmitt (Hrsg.), Handbuch Translation (S. 301-304). Tübingen: Stauffenburg Verlag.

Pöchhacker, F. (2004). Introducing interpreting studies. London-New York: Reutledge.

Pöchhacker, F. / Zwischenberger, C. (2010). Survey on quality and role: Conference interpreters' expectations and self-perceptions. http: //aiic.net/p/3405.

Prunč, E. (2007). Entwicklungslinien der Translationswissenschaft: Von den Asymmetrien der Sprachen zu den Asymmetrien der Macht. Berlin: Frank \& Timme.

Prunč, E. (2009). Was haben Chamäleons den TranslatorInnen zu sagen. In: H. Kalverkämper / L. Schippel (Hrsg.), Translation zwischen Text und Welt - Translationswissenschaft als historische Disziplin zwischen Moderne und Zukunft (S. 115-137). Berlin: Frank \& Timme.

Reiss, K. / Vermeer, H. J. (1984). Grundlegung einer allgemeinen Translationstheorie. Tübingen: Niemeyer.

Seleskovitch, D. (1968). L'interprète dans les conferences internationals. Paris: Lettres modernes Minard.

Tymoszuk, K. (2016). Przetwarzanie tekstu w ttumaczeniu symultanicznym. Lublin: Wydawnictwo Uniwersytetu Marii Curie-Skłodowskiej.

Venuti, L. (1995). The translator's invisibility: A history of translation. London-New Jork: Routledge.

Received: 07.08.2018; revised: 09.02.2019 